\title{
An Analysis of Diesel Flame by Picture Processing*
}

\author{
Masahiro SHIOJI**, Takashi KIMOTO**, \\ Masaaki OKAMOTO*** and Makoto IKEGAMI**
}

\begin{abstract}
A picture-processing techique is applied to the study of flame motion in a directinjection diesel engine. From high-speed photographs, two-dimensional cross-correlation coefficients of flame luminosity between two successive frames are obtained. The location and value of the maximum coefficients give us the mean and fluctuating velocities of the flame, respectively. The results show that the fluctuating velocity obtained in a wide region is almost independent of measuring position and size in the middle and later stages of combustion, indicating that the turbulent flow field is almost uniform and isotropic over the space in diesel engines. The effects of the swirl and chamber geometry on flame behavior are discussed in detail.
\end{abstract}

Key Words: Internal Combustion Engine, Diesel Flame, High-Speed Photograph, Picture Processing, Cross-Correlation, Swirl, Turbulence

\section{Introduction}

In the combustion process in diesel engines, turbulence is important because it plays a role in mixing fluid lumps having different fuel concentrations. Although several types of anemometry have been developed, it is so difficult to measure the in-cylinder gas motion during combustion that scarcely any realistic measurements have been made. This is because a large cyclic variation hinders the complete discrimination of the fluctuating velocity from the time-sequen. tial signal obtained by conventional anemometry.

With these facts in mind, high-speed photographs of diesel flames ${ }^{(1) \sim(3)}$ are analyzed to determine the distribution and motion of the luminous flamelets. A computer-aided picture-processing technique employing two-dimensional cross-correlation coefficients of flame luminosity between two successive

* Received 26 th October, 1988. Paper No. 87-1248 A

" Faculty of Engineering, Kyoto University, YoshidaHonmachi, Sakyo-ku, Kyoto, 606, Japan

** Toyota Automatic Loom Works, Ltd., 2-1 Toyotacho, Kariya, 448, Japan frames is used. The location and the value of the maximum coefficients represent the mean difference in position during a certain time interval and the intensity of random motion of the flame, respectively, and thus provide information on both bulk motion and turbulence. Unfortunately, since the luminosity fluctuation in direct photographs is used as the marker, the velocity obtained in the present study does not exactly agree with the fluid velocity, but gives the averaged group velocity of the flamelets on the path in the viewed direction. The picture at a single section may make it possible to determine the motion at a certain cross section.

This cross-correlation technique has been applied in attempts to find the distributions of velocity vectors in a steady flow ${ }^{(4)}$ and in a diesel engine combustion space $^{(5)}$. In the present paper, this technique is extended so as to yield information on turbulence. In addition, the effects of the swirl and chamber geometry on the behavior of the flame in a direct-injection diesel engine are discussed based on the results of picture analysis. 


\section{Photographs for Picture Processing}

\subsection{Experimental conditions and processing techniques}

In the present study, high-speed photographs of diesel flames ${ }^{(1)}$ which were taken by one of the present authors in a double-scavenged two-cycle single-cylinder test engine were used for the picture processing. Figure 1 shows the configuration for photographing combustion: A tempered-glass window was installed on the cylinder head to make it possible to observe the behavior of one of the five sprays. Photographs were taken for three swirl ratios in three types of combustion chambers having different bowl diameters, as shown in Fig. 2. The main specifications and operating conditions of the test engine are given in Table 1, and the photographic conditions are summarized in Table 2.

Table 1 Engine specifications and conditions

\begin{tabular}{l|l}
\hline \hline Cylinder bore, stroke & $110 \mathrm{~mm}$ and $120 \mathrm{~mm}$ \\
Compression ratio & $19.6: 1$ \\
Real compression ratio & $14.0: 1$ \\
Injection pump & Bosch PE2B 100 \\
Injection nozzle & Five $0.22 \mathrm{~mm}$-hole nozzle \\
Opening pressure & $24.5 \mathrm{MPa}$ \\
Spray angle & $140 \mathrm{deg}$ \\
Cetane number of fuel & $70 \mathrm{mg}$ per fired stroke \\
Fuel delivery & $37.2 \mathrm{mg}$. \\
Static injection timing & $20 \mathrm{deg}$. BTDC \\
\hline
\end{tabular}

Table 2 Conditions of photographic run

\begin{tabular}{|c|c|c|c|c|}
\hline Run No. & Chamber & $r_{s}$ & $\begin{array}{c}\text { Engine speed } \\
\text { rpm }\end{array}$ & $\begin{array}{l}\text { Camera speed } \\
\text { frames/sec }\end{array}$ \\
\hline 1 & I & 0 & 1090 & 5750 \\
\hline 2 & $I$ & 2.5 & 1270 & 6000 \\
\hline 3 & II & 0 & 1120 & 6060 \\
\hline 4 & I I & 1.8 & 1040 & 5420 \\
\hline 5 & II & 2.7 & 1150 & 6000 \\
\hline 6 & III & 0 & 1170 & 6080 \\
\hline 7 & III & 1.8 & 1230 & 5910 \\
\hline 8 & I I I & 2.7 & 1300 & 6250 \\
\hline
\end{tabular}

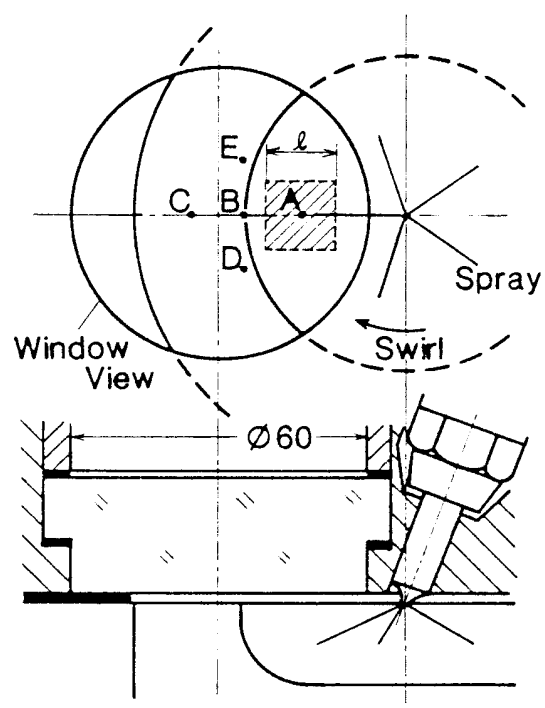

Fig. 1 Configuration for photographing combustion
To detect the flame luminosity from the film negative, the drum scanner at the Data Processing Center, Kyoto University (Optronics, C 4100-30) was used with a spatial resolution of $50 \mu \mathrm{m}$ on $16-\mathrm{mm}$ highspeed film. The intensity of the light penetrating through the film was locally detected and digitized to an 8-bit numerical value corresponding to a 256 resolution, which was normalized from zero to unity to define the intensity $I$. The digitized picture of each frame was processed to match the position sequentially using the rim of the window as a reference. Each picture was stored on magnetic tape to facilitate the analysis.

\section{2 Optical density variation of the photographs}

First, the photographs taken under a typical condition were processed to obtain the optical density variation or the spatial distribution of the diesel flames on the picture. Figure 3 shows examples of a gray-level picture for optical density obtained with the crank angle $\theta$ in a flat-bottomed deep-bowl-type chamber (Type II in Fig. 2) for three swirl ratios $r_{s}$ determined at the start of compression. The dark part of each picture represents the luminous flame. In Fig. 3 , the effects of swirl on the distribution of the flamelets are indicated: At $r_{s}=0$, a fan-shaped initial flame forms in the bowl center, spills off into the clearance space between the piston top and the head, and then disappears from the bowl center. On the other hand, an excessive swirl leads to poor combustion due to weak penetration of the flame into the clearance, and large lumps of flamelets remain in the bowl. A moderate swirl expands the flame over the combustion space in the relatively early stages of combustion.

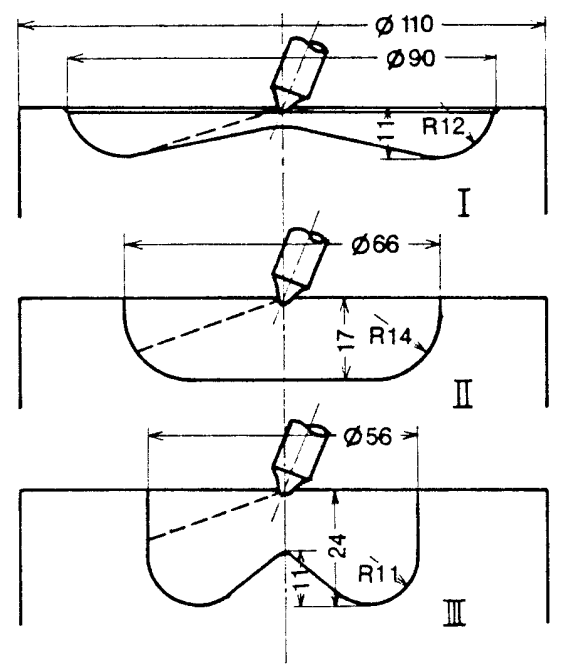

Fig. 2 Cross section of combustion chamber 
As shown, many lumps of flamelets having various sizes and different luminosities appear in the combustion space and change with time. They can be detected as the density fluctuation in a picture. Figure 4 (a) exemplifies the change of one-dimensional den- sity distributions in the $y$-direction, defined as the direction downstream of the swirl and vertical to the direction of fuel injection. These distributions are obtained at a distance from the cylinder axis and along the axis of injection of $x=24 \mathrm{~mm}$ for 5 succes-
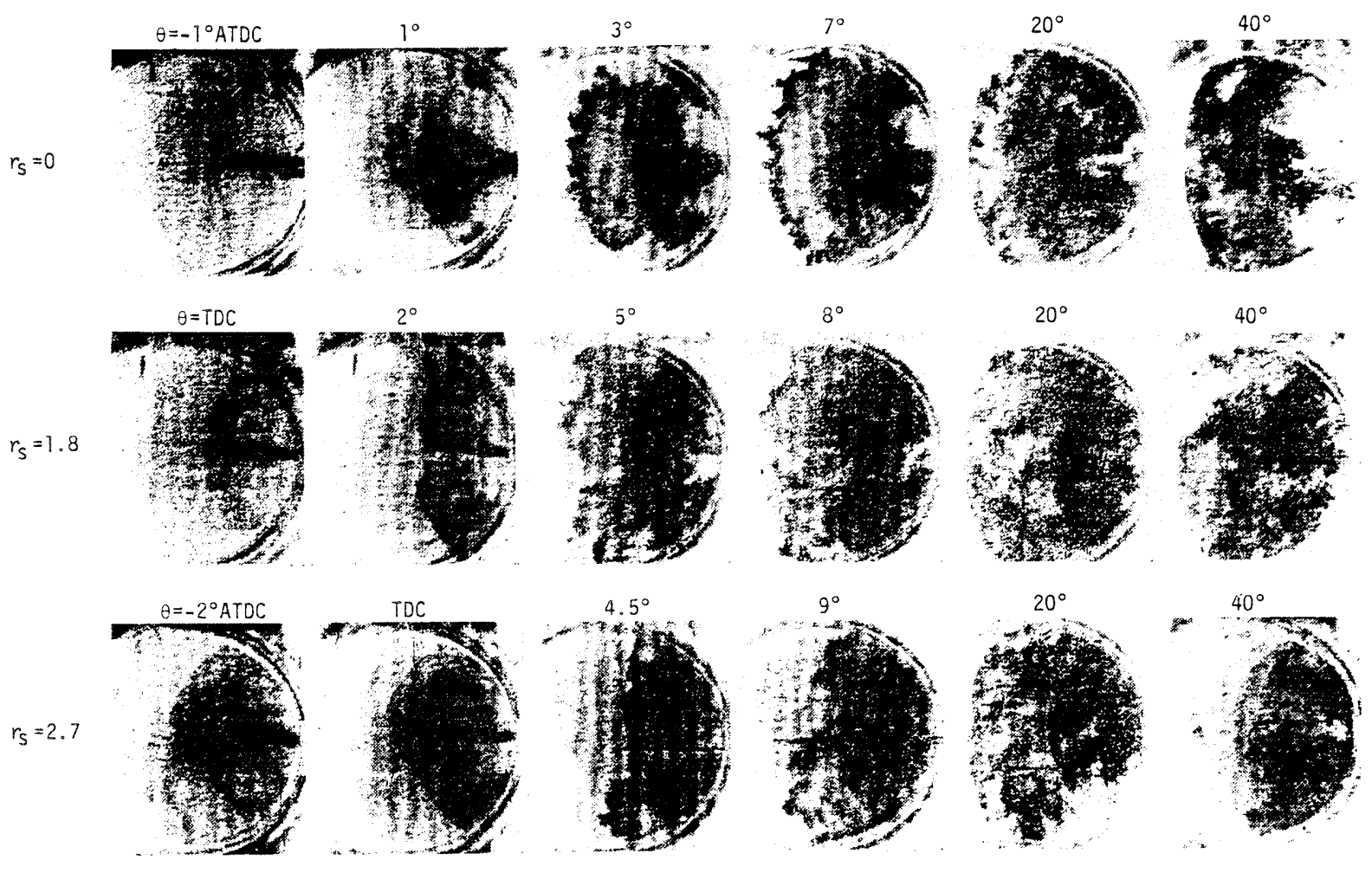

Fig. 3 Gray-level outputs of optical density variation of the photographic film

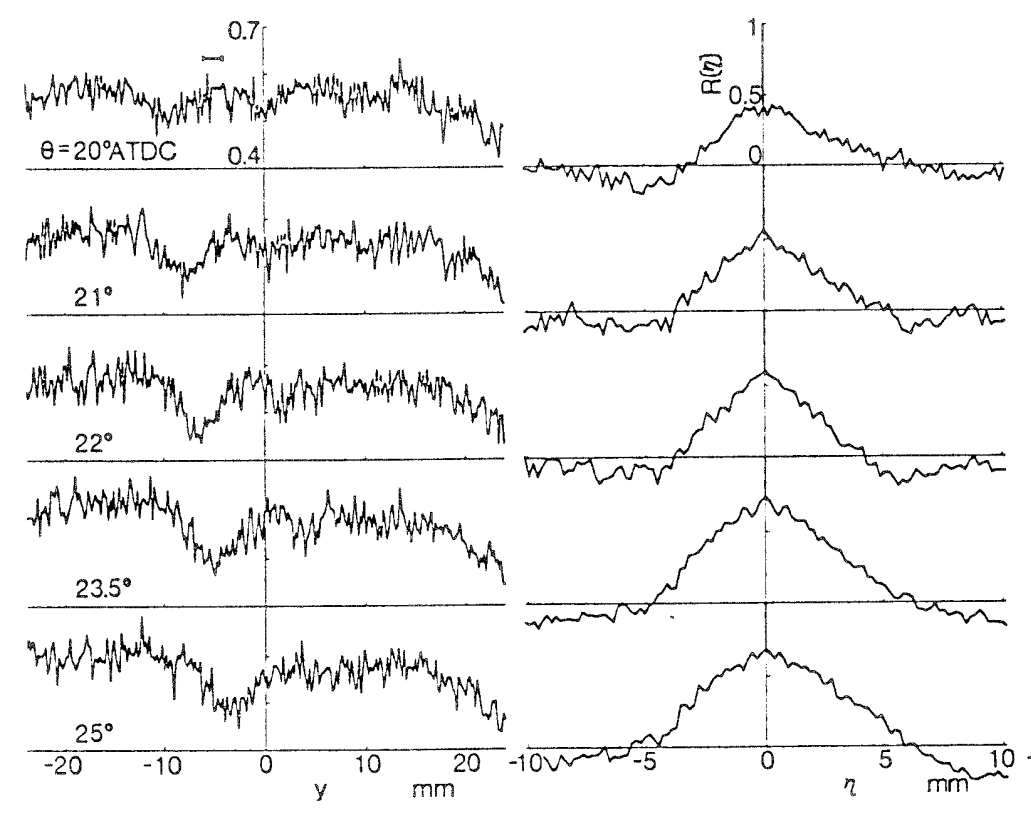

(a) (b)

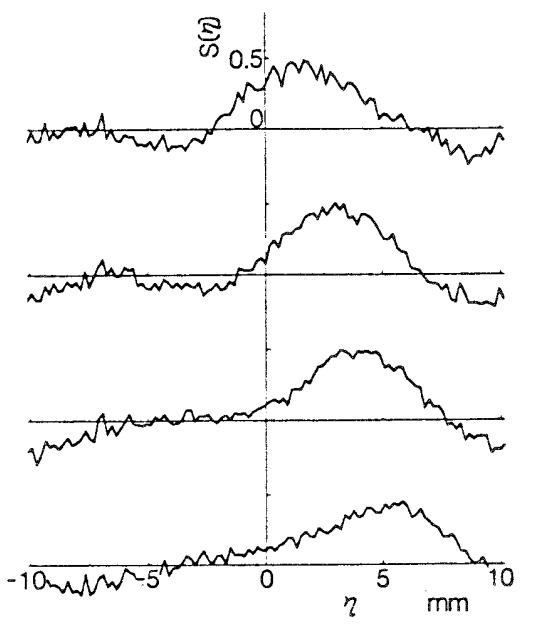

(c)

Fig. 4 One-dimensional optical density distribution $I$, autocorrelation coefficients $R$ and cross-correlation coefficients $S$ [Run No.4] 
sive frames of the pictures, with the swirl condition $r_{s}$ $=1.8$, as shown in Fig. 3 . Although the optical density distribution in each picture fluctuates randomly, it might be characterized by a statistical quantity over a certain view region. Figure 4 ( b ) indicates the spatial autocorrelation functions calculated from the density fluctuations of Fig. 4 (a) in the range of $-20 \mathrm{~mm}<y$ $<20 \mathrm{~mm}$. Each function decays with the spatial difference in the $y$-direction, $\eta$, and the spread represents the ensemble mean scale of the fluctuation of flame luminosity. In this figure, the spread of every autocorrelation function has an approximately constant value of $3 \mathrm{~mm}$. Therefore, rapid change of the flame structure may not occur under the experimental conditions pertaining to this figure.

Figure $4(\mathrm{c})$ shows the cross-correlation functions calculated between the optical density fluctuation of Fig. 4(a) at every crank angle and that at $\theta$ $=20^{\circ} \mathrm{ATDC}$ in the same region as in Fig. $4(\mathrm{~b})$. Each curve has a maximum whose position is remote from the origin and whose value decreases as the time interval between two pictures increases. Since the cross-correlation function has a maximum at the position where the density distributions of two images are most like each other, the maximum position clear. ly indicates the mean difference and direction of the flame moving at a certain time interval within the view region. In addition, a decrease in the maximum value indicates the degree of disagreement in the density distribution which may be attributed to turbulent random motion. In other words, such a decrease includes some information on turbulence.

This is the concept behind the technique employed in the present study. In the analysis, the flame motion on a plane is actually estimated from the two-dimensional cross-correlation function. Before this technique is extended to the estimation of turbulence, the following points should be discussed:

(1) Ability to locate the flame velocity at any depth from the cylinder head, where the observation of flame luminosity was made, was considered. Kamimoto et al. ${ }^{(5)}$ determined the flame motion by the cross-correlation of the luminosity detected simultaneously at different locations. They showed that after the initial stages of combustion, the velocity was detected at points within the combustion space rather than near the cylinder head since the soot concentration became lower over the whole of the combustion space. Accordingly, the velocity at a position well within the flames may be obtained during the middle and later stages of combustion by this type of optical method.

(2) The effect of the occurrence or disappearance of the flame on the velocity estimation was assessed from the variation in the PDF of optical density. Figure 5 indicates the PDFs obtained at a specified crank angle in Run No. 4 of Table 2, each of which is superimposed on that of the next frame. Just after ignition of $\theta=2^{\circ} \mathrm{ATDC}$, a remarkable change in PDF occurs, accompanied by the rapid expansion of the flame into the combustion space. This change apparently results in an increase in the velocity in the direction of the flame occurrence. After this, however, the PDF scarcely changes during the short period between frames. This suggests that the effect of flame occurrence on the velocity estimation can be disregarded, except for in the very early stages of combustion.

(3) The effect of the spatial difference in velocity on the estimation of turbulence was considered. In a case in which the flame motions at different cross sections are detected on a plane, the velocity differences may be overlapped and probably lead to overestimation of the intensity of turbulence. Fortunately, as we shall see, the variation in the density distribution may be assumed to be uniform and isotropic in the main stages of combustion, suggesting that a convective flow whose velocities differ in the upper and lower layers does not affect the volumeweighted average of the fluctuating velocity as long as the direction of the main flow is uncorrelated with turbulent motion. Therefore, the rms intensity of turbulence can be estimated by averaging in a large region.

(4) In order to calculate the correlation function, it is necessary to determine the size of the processing region. Figure 6 presents the contours of the two-

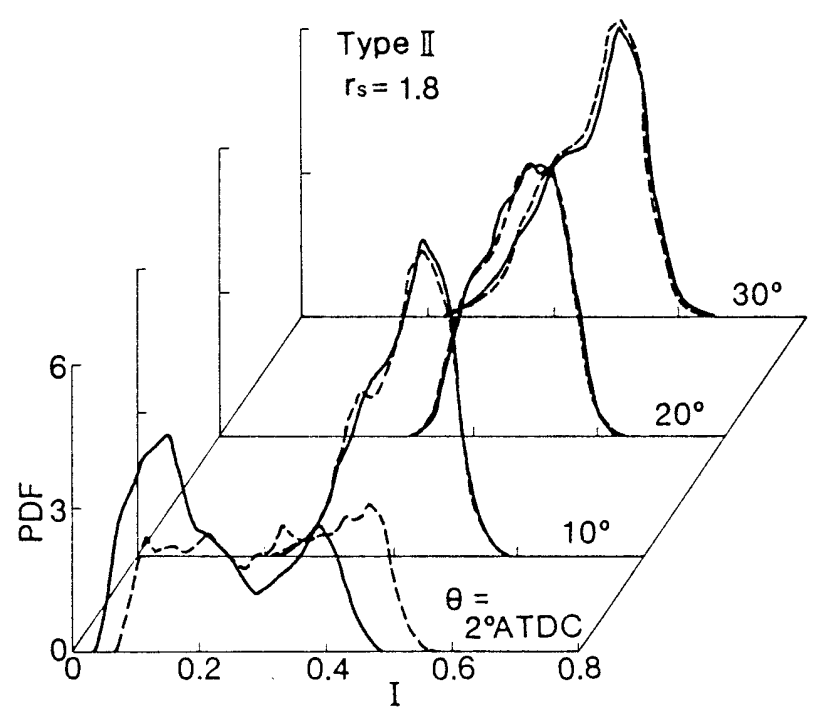

Fig. 5 Variation in PDF of optical density of two successive pictures 
dimensional cross-correlation coefficients calculated from the picture at $\theta=20^{\circ} \mathrm{ATDC}$ in Run No. 4 at point $B$ (See Fig. 1) for different sizes of a square window having a side $l$. When $l$ is small, the coefficient exhibits a complicated distribution of an oscillating and random nature. When $l$ is greater than $10 \mathrm{~mm}$, there is a definite maximum at every point, as shown at point B. In other words, to use the luminous flamelets as the tracer, more than twice the region of the typical scale of the density fluctuations should be selected. Once this condition is satisfied, the maximum of the two-dimensional cross-correlation function of two pictures gives the volume-weighted average motion of the flame over the selected region during the time interval.

\section{3 Cross-correlation function of the pictures}

Based on the discussion above, the relationship between the maximum of the cross-correlation function calculated from two pictures and the movement of the flame is investigated. Let $I(t, x, y)$ be the optical density distribution function at time $t$, and express the cross-correlation function of the image after time interval $\Delta t$ by the following equation:

$$
\begin{aligned}
& S(\xi, \eta, \Delta t)= \\
& \frac{\left[\begin{array}{c}
\langle \\
\{I(t, x, y)-\langle I(t)\rangle\} \\
\times\{I(t+\Delta t, x+\xi, y+\eta)-\langle I(t+\Delta t)\rangle\}\rangle
\end{array}\right]}{\left[\begin{array}{c}
\sqrt{\left\langle\{I(t, x, y)-\langle I(t)\rangle\}^{2}\right\rangle} \\
\left.\times \sqrt{\left\langle\{I(t+\Delta t, x+\xi, y+\eta)-\langle I(t+\Delta t)\rangle\}^{2}\right\rangle}\right]
\end{array}\right.}
\end{aligned}
$$

where $\xi$ and $\eta$ denote the distances in the directions of $x$ and $y$, respectively, and $\langle>$ represents the ensem. ble average in the calculation region. If the density
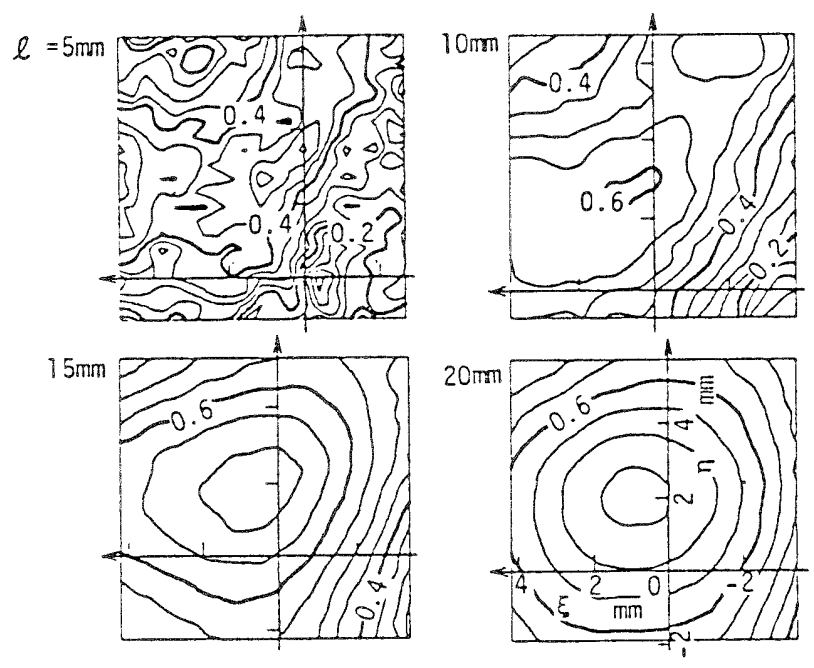

Fig. 6 Contour of two-dimensional cross-correlation coefficients for different sizes of square window [Run No.4, at Point B in Fig. 11] distribution of one image has no correlation with the other, $S$ tends to be zero. If the two images are identical, $S$ equals the autocorrelation function $R=$ $S(0, \xi, \eta)$. In a case in which the flame moves by convection, $S$ has a maximum at the same positions of $\xi$ and $\eta$ as the magnitude of the movement of the flame, thus giving the velocity vector averaged in the calculated region. Otherwise, when the density distribution is changed by random flame motion due to turbulence, the maximum value of the coefficients decreases with the intensity of such motion. If this decrease is predicted as a function of turbulence, it may be possible to estimate turbulence by comparing the decrease obtained from the actual pictures with the predicted decrease. From this point on, the probability of the movement of fluid elements will be consid. ered to predict the density distribution of the next picture, as in the Fokker-Planck equation.

Now suppose that a fluid element having a density $I$ at the position $(x, y)$ at time $t$ moves on the flow to the position $(x+\Delta x, y+\Delta y)$ during the time interval $\Delta t$. Let the probability of its motion be $p(\Delta x, \Delta y)$, and let the density distribution after $\Delta t$ be

$$
\begin{aligned}
& I(t+\Delta t, x, y)=\iint_{-\infty}^{\infty} d \Delta x d \Delta y \\
& \quad \times I(t, x-\Delta x, y-\Delta y) p(\Delta x, \Delta y)
\end{aligned}
$$

After substitution into Eq. (1), we obtain

$$
\begin{aligned}
& S(\xi, \eta, \Delta t)=\iint_{-\infty}^{\infty} d \Delta x d \Delta y R(\xi-\Delta x, \eta-\Delta y) \\
& \quad \times p(\Delta x, \Delta y)
\end{aligned}
$$

This equation indicates that once $p(\Delta x, \Delta y)$ is defined, $S$ can be predicted from $R$ at time $t$. Since the global motion of the element may not affect the maximum value of $S, S_{0}$, it is sufficient in describing $p(\Delta x, \Delta y)$ to consider only the change due to turbulence. For this, we assume that every fluid element moves randomly

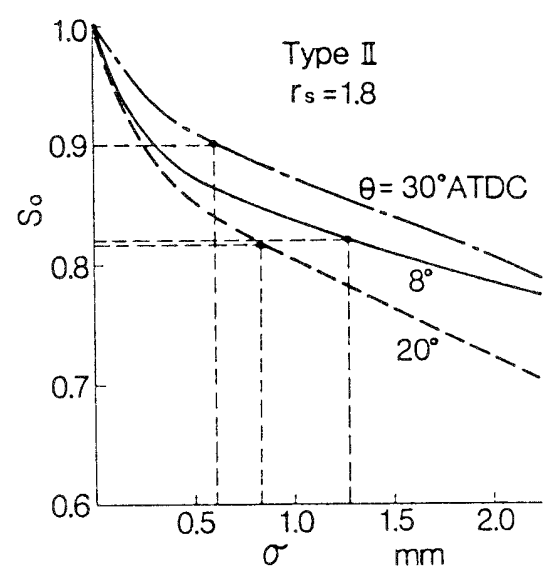

Fig. 7 Changes of the predicted $S_{0}$ against $\sigma$ [at point $\mathrm{B}, l=30 \mathrm{~mm}$ ] 
in every direction on a plane, and that the probability of its motion is described by the following two-dimensional isotropic Gaussian distribution function :

$$
p(\Delta x, \Delta y)=\exp \left\{-\left(\Delta x^{2}+\Delta y^{2}\right) / 2 \sigma^{2}\right\} /\left(2 \pi \sigma^{2}\right)
$$

where $\sigma$ denotes the standard deviation of the distribution. Figure 7 shows the predicted change of $S_{0}$ against an assumed $\sigma$ for the picture having $r_{s}=1.8$ in Fig. 3. $S_{0}$ decreases exponentially with $\sigma$. Comparing the maximum cross-correlation coefficient obtained from the actual photographs with the predicted one and finding the fit point, as shown in Fig. 7, we can determine the most probable standard deviation.

\section{4 Estimation of turbulence}

If the random motion of the element is equivalent to that due to turbulence, $\sigma$ gives the width of the statistical spread of one-particle turbulence diffusion $^{(6)}$. From this point on, the relationship between $\sigma$ and the intensity of turbulence should be investigated. Since the turbulent motion consists of eddies having various scales, $\sigma$ is expected to be significantly dependent upon the calculated region. Figure 8 shows the changes of $\sigma$ against the side of the square-window region, $l$, for several crank angles $\theta$ at point $B$ in the

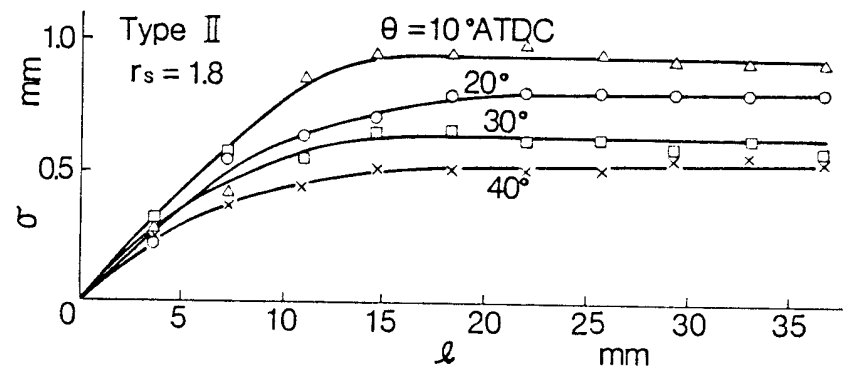

Fig. 8 Changes of $\sigma$ with $l$

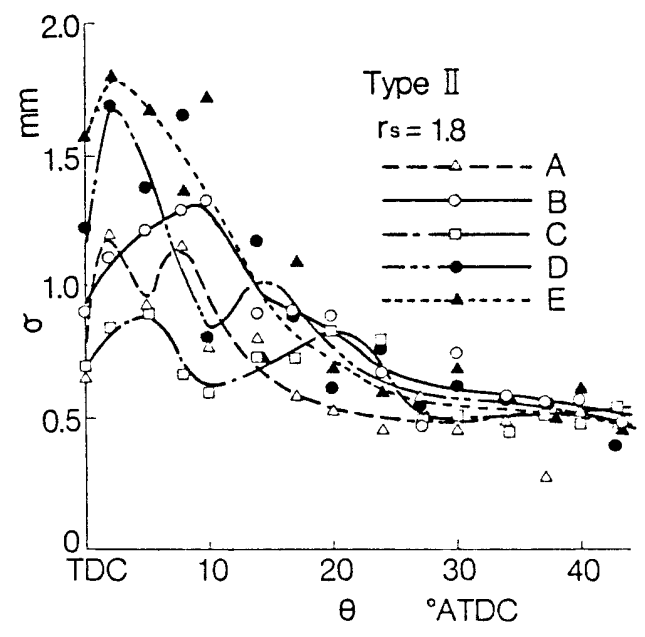

Fig. 9 Courses of $\sigma$ at different positions pictures of Run No. 4. In every curve, $\sigma$ first increases with $l$, then saturates, and tends toward a constant for a large $l$. This indicates that the fluctuating motion of a fluid element is restricted by the distance between each pair of elements, as well as by the turbulent motion. In Fig. 8, it is shown that the intensity of the fluctuating motion can be obtained for a short time difference with an $l$ greater than $20 \mathrm{~mm}$.

As shown above, this technique is suitable for the analysis of flame motion in unsteady turbulent flow fields because it requires only two pictures for a short duration. Otherwise, the volume average should be performed in a relatively large region; thus, the values obtained at different positions should not be largely altered. To ascertain the uniformity of $\sigma$, estimations were made at different positions. Figure 9 shows time variation obtained at a window size $l$ of $30 \mathrm{~mm}$ for a typical condition at the five positions indicated in Fig. 1. Just after ignition, $\sigma$ varies from position to position. However, in the middle and later stages of combustion, this value is almost independent of the measuring position, whether in the bowl or the clearance space. This suggests that the random flamelet motions are almost uniform and isotropic over the entire range of observation.

Another problem remaining in calculating the cross-correlation is the time interval between two pictures. If this interval is too long to hold the correlation of the density distribution of flamelets, the present technique may no longer be applied. Figure 10 shows the changes in $\sigma$ estimated for several intervals $\Delta t$. In the calculation, one picture was fixed at a certain crank angle $\theta$ and another one was shifted frame by frame. At every $\theta, \sigma$ first increases proportionally with $\Delta t$, but the rate of increase later reduces with $\Delta t$. This relation between $\sigma$ and $\Delta t$ may be well explained by the one-particle diffusion theory in a uniform and isotropic turbulent flow, in which the diffusion width is equal to the product of the diffusion time $T$ and the intensity of turbulence $u^{\prime}$ for small $T$ and proportionally increases with $\sqrt{T}$ for large $T$. In

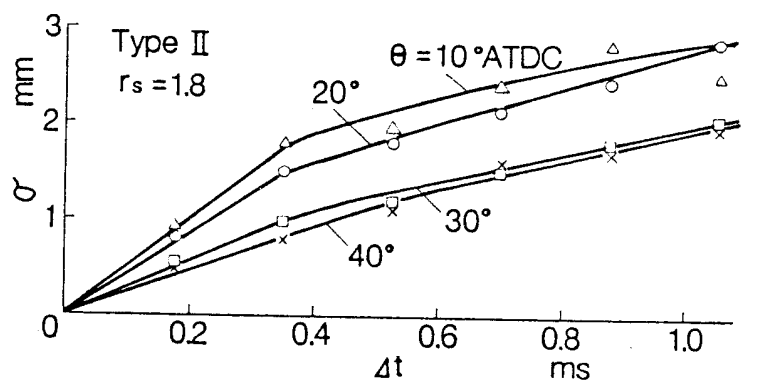

Fig. 10 Changes of $\sigma$ with $\Delta t \quad$ [at point B, $l=30 \mathrm{~mm}$ ] 

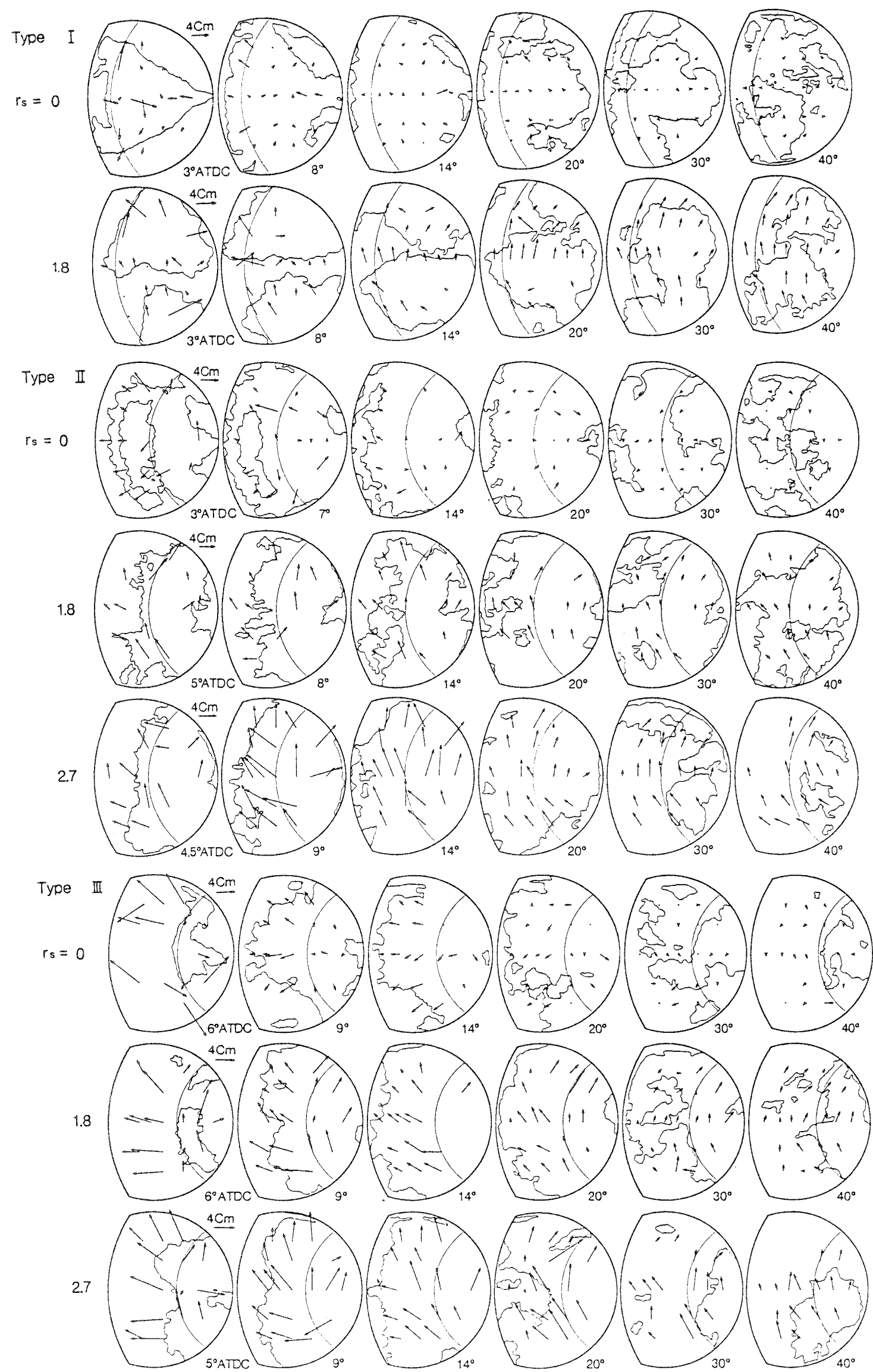

Fig. 11 Velocity vectors of flame $\left[C_{m}\right.$ denotes mean piston speed] 
other words, the time interval $\Delta t$ between adjacent pictures is sufficiently short, and as a result the intensity of turbulence may readily be determined by dividing the obtained $\sigma$ by $\Delta t$.

\section{Gas Flow in Diesel Flame}

As shown above, the mean velocity and the intensity of turbulence can successfully be estimated from the shift and the value of maximum cross-correlation coefficients between two successive frames. The velocity obtained from luminosity fluctuation in direct photographs does not exactly agree with the fluid velocity. Nevertheless, it may be plausible to assume that they are equal since the flame motion essentially tracks the flow, except in the very early exploding phase.

\subsection{Orderly flame motions}

Figure 11 maps the velocity vectors determined by this method using a square window having $10 \mathrm{~mm}$ sides. They were obtained at various positions for three types of combustion chambers, shown in Fig. 2, with and without swirl. The profile of the flaming zone is sketched in each figure, with the constant film concentration line leveled to a maximum density before ignition. In the earlier stages of combustion, the flame motions appear to be random in direction at high speeds, indicating fast flame expansion due to ignition. In the middle and later stages of combustion, it is noted that the flame scarcely moves in the absence of swirl, but is very active when swirl is present. In particular, the flame moves to the cylinder center in the bowl and to the outer region in the clearance. The velocity of such flame motions increases once just after reaching top dead center. This acceleration is more remarkable in the combustion chamber with the smaller diametral ratio of the bowl to the cylinder, $d / D$. After reaching a crank angle $\theta$ of $20^{\circ} \mathrm{ATDC}$, the flame mainly moves in a tangential direction on the swirl rather than in a radial one.

In the deep-bowl-type chambers (Types II and III), the tangential velocity increases with crank angle during the diffusive burning period, suggesting that fuel-air mixture expansion occurring in the tip of the spray greatly accelerates the flow. After such a large increase in velocity, the swirling flow gradually decays with crank angle. These observed effects of swirl on the flame velocity are almost consistent with the measured results of Kamimoto et $\mathrm{al}^{(5)}$. In addition. it is noted that change of the flame motion by a swirl is also remarkable at a small $d / D$. By contrast, in the shallow-dish chamber (Type I), the tangential velocity increases only slightly after ignition and does not decrease, even in the later burning stages.

\section{2 Intensity of turbulence in a flame}

Next, based on the discussion in section 2.4, we obtain the intensity of turbulence $u^{\prime}$ for a sufficiently large $l(30 \mathrm{~mm})$. In Fig. 12, the changes in $u^{\prime}$ with crank angle are shown under the same conditions as in Fig. 11. Also indicated are the changes in average intensity $\langle I\rangle$ calculated at each picture. In every case, $u^{\prime}$ increases with crank angle just after ignition or in the middle stages of combustion, and then gradually decreases. The increase in $u^{\prime}$ during combustion indicates that the random motion of the flame is heavily activated by the combustion, which greatly

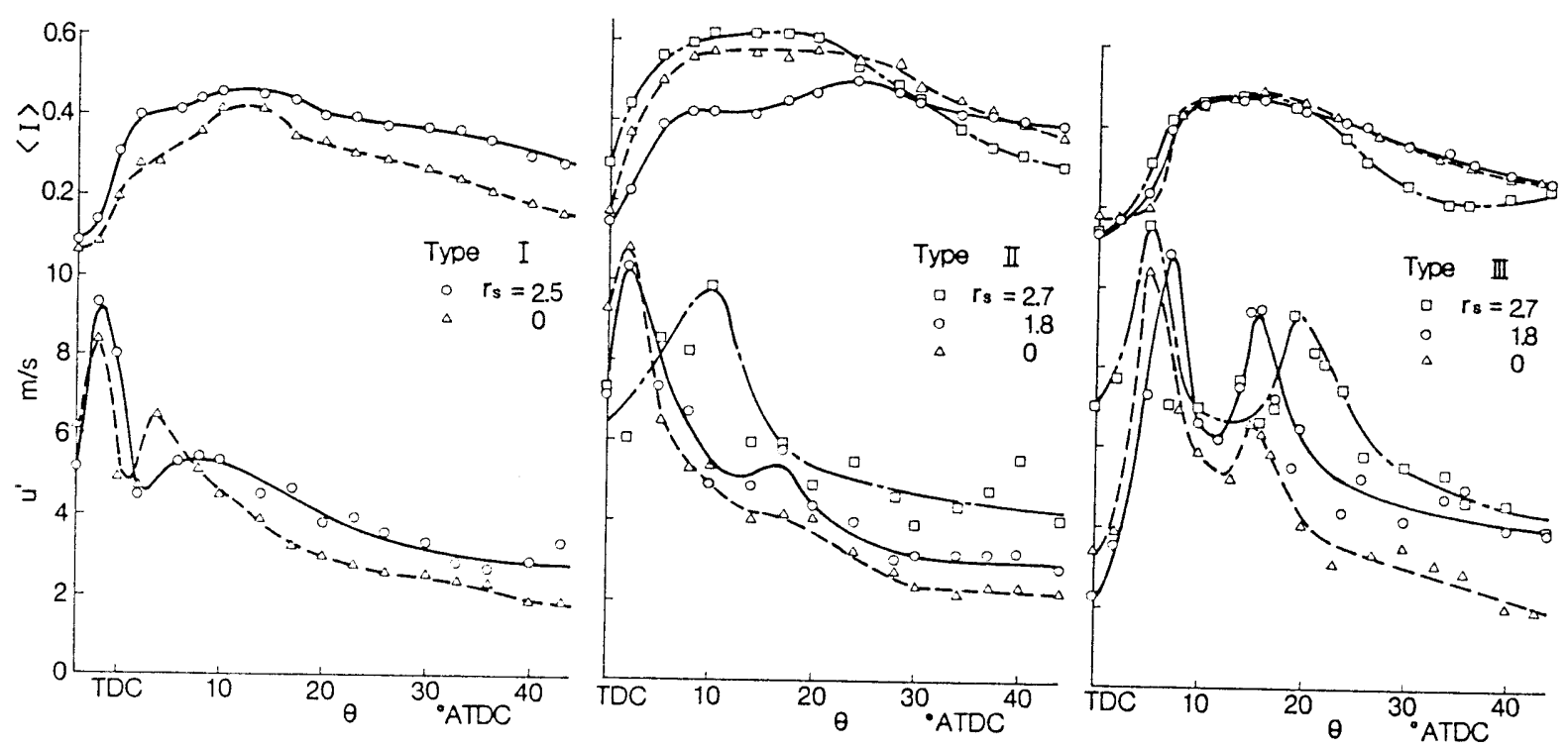

Fig. 12 Courses of average intensity of turbulence $u^{\prime}$ and average flame luminosity $\langle I\rangle$ under various conditions 
depends on both the swirl condition and the shape of the combustion chamber.

In the shallow-dish-type chamber, $u^{\prime}$ rapidly decreases with crank angle and reaches $2 \sim 3 \mathrm{~m} / \mathrm{s}$ in the last stage of combustion, irrespective of the swirl ratio. By contrast, in the deep-bowl chamber (Types II and III), the decay of $u^{\prime}$ is much delayed at a high swirl ratio. It is particularly noteworthy that $u^{\prime}$ increases again in the middle stages of combustion at a small $d / D$. In this case, $u^{\prime}$ remains at a high level until later stages.

From comparison with the results in Fig. 11, it may be pointed out that the condition in which the decrease in tangential velocity is much delayed yields a large $u^{\prime}$. This suggests that a swirl may generate turbulence and thereby promote fuel-air mixing in the later burning stages. Otherwise, in the shallow-dishtype chamber, it might be necessary to enhance turbulence using some other methods instead of swirl. One possibility along this line is to increase the fuel-injec. tion pressure to cause a much higher shear stress in the spray; another is to require a projection in the combustion space so as to make small eddies behind it by interfering with the gas stream.

Two of the present authors proposed a stochastic model $^{(7)}$ for predicting diesel combustion in which flame-generated turbulence in the heterogeneous density field was suggested. This concept is quite consistent with the results obtained in the present study. However, the volume-averaged turbulence estimated from flame motion cannot be quantitatively compared with the mass-averaged turbulence predicted by the model. This requires further clarification.

\section{Conclusions}

The present study proposed a new picture-processing technique which made it possible to estimate the averaged velocity and intensity of turbulence in directinjection diesel engines from high-speed photographs of the flame. This technique was based on the assumption that change in the optical density variation of photographic film depends upon fluid motion. Both mean movement and change in the distribution of luminosity fluctuation over the specified range of observation were statistically obtained using the maximum of the cross-correlation function of two pictures. From the results obtained under a typical condition, bulk motion could be determined at more than twice the region of the average scale of the density fluctuation. For a region of more than a certain size, the fluctuating velocity obtained was independent of measuring position and size. This sug- gests that the turbulent flow field is almost uniform and isotropic in the main parts of combustion; therefore, the intensity of turbulent motion can be estimated.

Using this method, the effects of the swirl and chamber geometry on flame behavior were investigated. From the analysis, the following results were obtained: Without swirl, the flame scarcely moved after the middle stages of combustion. In the case in which swirl was present, and when a deep-bowl-type combustion chamber was used, the tangential velocity increased with crank angle. At the same time, the random motion of the flame was activated and the intensity of turbulence increased significantly. In particular, at a small diametral ratio of the bowl to the cylinder, the tangential velocity rapidly decreased after once increasing. In such a case, the turbulence remained at a high level even in the later burning stages. This suggests that a swirl may generate turbulence and thereby promote fuel-air mixing in the later stages of combustion.

This study was partially supported by a Grant-in Aid for Scientific Research (No. 61460106) received in 1987 from the Ministry of Education, Science and Culture of Japan.

\section{References}

(1) Nagao, F., Ikegami, M., Kiyota, Y. and Fujikawa, T., A Study on Combustion in Direct-Injection Diesel Engines, Bull. JSME, Vol. 16, No. 93 (1973), p. 588 and 599 .

(2) Alcock, J. F. and Scott, W. M., Some More Light on Diesel Combustion, Proc. Inst. Mech. Eng. (A. D.), Vol. 5 (1962-63), p. 179.

(3) Nishida, K., Murakami, A. and Hiroyasu, H., Measurements of Swirling Flow in a Direct Injection Diesel Engine Under Motored and Fired Conditions, Proc. 20 th Symp. Int. Combust. (1984), p. 201. The Combustion Institute.

(4) Kimura, I., Takamori, T. and Inoue, T., Image Processing Instrumentation of Flow Velocity Vector Distribution Using Correlation Techique, (in Japanese), Trans. Soc. Instr. \& Contr. Eng., Vol. 23, No. 2 (1987), p. 101.

(5) Kamimoto, T., Miyairi, Y., Nagakura, K. and Matsuoka, S., Measurement of Flow Velocity in Diesel Flames by a Cross-Correlation Method, SAE Paper No. 820357 (1982).

(6) Hino, M., Fluid Dynamics (in Japanese), (1974), p. 235, Asakura-Shoten.

(7) Ikegami, M., Shioji, M. and Koike, M., A Stochastic Approach to Model the Combustion Process in Direct-Injection Diesel Engines, Proc. 20 th Symp. Int. Combust., (1984), p.217, The Combustion Institute. 